\title{
Colombia: Perfil de salud reproductiva 1990
}

\author{
Dr. Oscar Ivan Rojas R.
}

Centro Colaborador OMS, Cali*

RESUMEN: COLOMBIA, perfil de salud reproductiva 1990. Se presenta una revisión actualizada de la información disponible sobre Salud Reproductiva en Colombia. E1 análisis se centra en identificar los cambios ocurridos y las tendencias de los indicadores durante los últimos 20 años. La creciente urbanización, el aumento de la población de mujeres en edad fértil, el permanente incremento en la tasa de uso de método anticonceptivos entre las mujeres de edad fértil y en unión en los últimos 20 años, son hechos sobresalientes. El documento señala áreas críticas, según se desprende del lento descenso de las tasas de mortalidad infantil, mortalidad materna , creciente abandono de la lactancia materna y tasa aparentemente elevadas de aborto en el país, especialmente en población adolescente. Como consecuencia del análisis se sugieren algunas líneas de intervención.

PALABRAS CLAVES: Cambios y tendencias de los indicadores de salud reproductiva en Colombia en los últimos 20 años: mortalidad materna, tasa de uso de métodos anticonceptivos en mujeres en edad fértil, prevalencia de amamantamiento, variaciones de la mortalidad infantil, aborto en adolescentes.

SUMMARY: Colombia, 1990 Health reproductive profile. An updated review of information available on Reproductive Health in Colombia is presented. The review and subsequent analysis are focused on the identification of changes and trends of the main indicators for the country in the last 20 years.

The continuing urbanization process, the rise in the percentage of female population in fertile age a long with the steady increase in the number of exposed women using contraceptive methods for the last 20 years, are all outstanding features of the Colombian reproductive profile. The paper underlines critical areas such as the slow decline of both maternal and infant mortality rates as compared to the statuts of the same indicators in other Latin American countries of similar level of development as Colombia. Attention is called upon the deleterious effect of the abandonment of nursing practices in lactating women and the apparent excess of abortion rates amongst adolescent women.

Areas of intervention are suggested as a result of the analysis.

KEY WORDS: Changes and trends in indictors of Reproductive Health in Colombia in the last 20 years, e.g.: maternal mortality, prevalence of use of contraceptive methods, infant mortality rate, prevalence of women practicing breastfeeding, abortion rates, etc.

Durante las últimas tres décadas Colombia ha experimentado profundos cambios en la estructura de su población (Cuadro 1).

De los 17 millones de habitantes en 1964, 4 millones $(22,6 \%)$ eran mujeres en edad reproductiva (15 a 49 años); para 1973 ya eran 5 millones ( $24 \%$ de la población), y para 1985 siete y medio millones de mujeres en ese grupo de edad representaban el $26,8 \%$ de la población total y el $53 \%$ de todas las mujeres. Paralelamente a estos cambios, la población menor de 5 años descendía de $17,6 \%$ del total de la población en 1964 a $14,3 \%$ en 1973 , y a $12,1 \%$ en 1985. En 1964 las mujeres mayores de 60 años (460.018) constituían el 2,6\% de la población total; en 1985 su número se duplicó (860.647) y pasaron a ser el $3.1 \%$ de la población total.

No sólo la distribución por edad ha cambiado en Colombia durante los últimos tres censos, también la distribución espacial ha sufrido profundas modificaciones. Para 1964, 5 millones de colombianos vivían en ciudades de más de

* I Seminario Taller de Identificación de Necesidades de Investigación en Reproducción Humana en Colombia. AA.: 6178 Cali-Colombia.

\section{Cuadro 1}

CAMBIOS EN LA ESTRUCTURA DE LA POBLACION COLOMBIANA, CENSOS DE 1964, 1973 y 1985

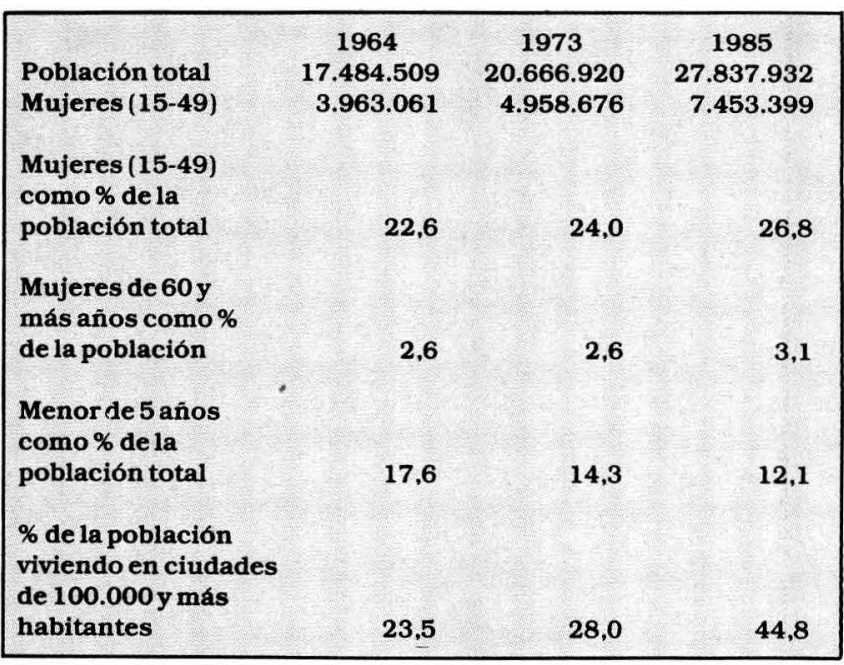

Fuente: DANE, censos 1964, 1973, 1985. 
100:000 habitantes (23\% de la población total); en 1985 la cifra había aumentado a 13 millones de habitantes (49\% de la población total).

Pese a la evidente modernización del país, en 1985 el $21 \%$ de las viviendas carecían de energía eléctrica, el $30 \%$ carecían de acueducto y el $48 \%$ no tenían alcantarillado. Departamentos con una altísima participación en el P.I.B. como Antioquia (15\%), carecían de electricidad en el $17,6 \%$, acueducto en el $26,9 \%$ y alcantarillado en el $31,4 \%$ de las viviendas. En departamentos con una baja participación en el P.I.B. como Caquetá $(0,46 \%)$, el $63 \%$ de las viviendas no tenían electricidad, el $54,5 \%$ no tenían acueducto y el 59,6\% no tenían alcantarillado. La tasa de analfabetismo en mayores de 15 años para Antioquia en el mismo censo se encontraba en $9,9 \%$, mientras que para Caquetá era del $16,4 \%$ y para el país de $12,2 \%$.

El comportamiento de los indicadores de salud entre 1970 y 1986 (Cuadro 2), nos muestra un moderado descenso de la mortalidad general, acompañado de cambios paralelos en la mortalidad infantil, que por su lentitud, dejan ver su dependencia de las deficientes condiciones sanitarias mencionadas antes.

Cuadro 2

COLOMBIA: INDICADORES DE SALUD 1970-1986

\begin{tabular}{|c|c|c|c|c|}
\hline & $1970-74$ & $1974-78$ & $1978-82$ & $1982-86$ \\
\hline $\begin{array}{l}\text { Tasa de mortalidad } \\
\text { general }\end{array}$ & 37.2 & 30.4 & 27.9 & 25.0 \\
\hline $\begin{array}{l}\text { Tasa de mortalidad } \\
\text { infantil }\end{array}$ & 70.6 & 65.0 & 60.0 & 55.4 \\
\hline Tasa de fecundidad & 4.6 & 3.8 & 3.5 & 2.0 \\
\hline $\begin{array}{l}\text { Expectativa de vida } \\
\text { al nacer }\end{array}$ & 61.5 & 62.0 & 62.5 & 63.5 \\
\hline$\%$ de analfabetismo & 19.4 & 16.7 & 14.9 & 12.8 \\
\hline $\begin{array}{l}\text { Tasa de crecimiento } \\
\text { del gasto público } \\
\text { en salud }\end{array}$ & 7.9 & 4.5 & 3.0 & 2.4 \\
\hline $\begin{array}{l}\text { P.I.B. por habitante } \\
\text { US\$ } 1970\end{array}$ & 598 & -- & -- & 824 \\
\hline $\begin{array}{l}\% \text { de las viviendas } \\
\text { en la cabecera }\end{array}$ & 58.4 & -.. & --. & 67.2 \\
\hline
\end{tabular}

Fuente: Ministerio de Salud. "Programa Materno Infantil: Hechos y Proyecciones", 1987.

La ganancia de sólo dos años en la expectativa de vida, en un período de 15 años, refleja la lentitud en el descenso de la mortalidad infantil en comparación con países de la misma región. Costa Rica entre 1970 y 1980 ganó 7,2 años en expectativa de vida $(65,4$ años en 1970 a 72,6 años en 1980) y su tasa de mortalidad infantil descendió de 57 por mil a 21 por mil en el mismo período (1).

El marcado descenso en la tasa de crecimiento del gasto público en salud y la lentitud en el descenso de las tasas de analfabetismo indudablemente contribuyen al estancamiento de las condiciones de salud en el período analizado. En
1985 el $39,5 \%$ de los hogares censados tenían sus necesidades básicas insatisfechas, y el 18,3\% (960.042 hogares) se encontraban en condiciones de miseria (2).

Al contrario de las tasas arriba analizadas, la tasa de fecundidad muestra un descenso rápido, comparable ahora sí con el de Costa Rica, a pesar de las diferencias en el modelo de desarrollo seguido por los dos países. Aunque las condiciones de urbanización creciente, el aumento del nivel educativo y el mejoramiento de las condiciones de salud son similares en otros países de la región, Colombia es uno de los pocos países que ha logrado un descenso tan notorio en sus tasas de fecundidad $(3,5)$. Los cambios observados en la tasa de fecundidad concuerdan con los descensos en la tasa bruta de natalidad (Cuadro 3), y con el promedio de hijos por mujer (Cuadro 4).

$$
\text { Cuadro } 3
$$

TASA BRUTA DE NATALIDAD EN COLOMBIA (POR MIL HABITANTES) SEGUN ESTIMACIONES DE VARIOS AUTORES

\begin{tabular}{|cccc|}
\hline \multicolumn{3}{c|}{ Fuente } \\
\hline Período & DNP & N.U.* & J. RICO \\
$1973-78$ & 30.4 & 33.3 & 32.8 \\
$1983-88$ & 27.9 & 31.0 & 30.4 \\
$1998-00$ & 20.4 & 24.3 & 21.5 \\
\hline
\end{tabular}

* (1970-75, 1980-85, 1995-00).

Fuente: Ministerio de Salud - Organización Panamericana de la Salud. Colombia: Diagnóstico de Salud, Políticas y Estrategias, 1984.

Cuadro 4

PROMEDIO DE HIJOS POR MUJER

\begin{tabular}{|lcccc|}
\hline IRegión & $\mathbf{1 9 5 1}$ & $\mathbf{1 9 6 4}$ & $\mathbf{1 9 7 3}$ & $\mathbf{1 9 8 5}$ \\
\hline Atlántica & 4.5 & 5.2 & 5.2 & 3.6 \\
Oriental & 4.8 & 5.9 & 5.9 & 3.1 \\
Central & 4.7 & 5.5 & 5.5 & 3.3 \\
Pacífica & 4.4 & 5.3 & 5.3 & 3.4 \\
\hline
\end{tabular}

Fuente: DANE, censos 1951-1985.

Otros determinantes de la fecundidad como son la edad promedio al matrimonio ( 22,1 años en 1976 y 23,6 años en 1986) y la duración de la lactancia (9 meses en 1976 y 11 meses en 1986), no son suficientes para explicar el cambio de 4,7 a 3,3 en la tasa global de fecundidad en el período analizado.

El incremento en el uso de métodos anticonceptivos, de $12 \%$ en mujeres en algún tipo de unión 1969 a $65 \%$ en 1986, es el componente más importante de dicho descenso. Del total de mujeres en algún tipo de unión en 1969, el $48 \%$ utilizaban anticonceptivos orales, $2,7 \%$ el DIU y $1,4 \%$ la esterilización. Para 1976 el 13,3\% eran usuarias de la píldora, $8,5 \%$ del DIU y $4,2 \%$ empleaban la esterilización. En 1986 (Figura 1), el método que más aumentó fue la esterilización, que pasó del 4,2\% en 1976 a 17,8\% en 1986.

Desafortunadamente la velocidad con que desciende la tasa global de fecundidad en el período 1980-1986 es sólo del $8 \%$ (3,6 a 3,3 hijos por mujer), comparada con un descenso del $43 \%$ entre 1969 y 1978 , cuando el promedio de hijos por mujer pasó de 6,7 a 3,8. Si miramos a las tasas de fecundidad por departamentos (Cuadro 5), encontramos grandes diferencias entre regiones del país que tienen grados diferentes de desarrollo. 
Figura 1

COLOMBIA. CAMBIOS EN LA PRACTICA ANTI-CONCEPTIVA 1969-86

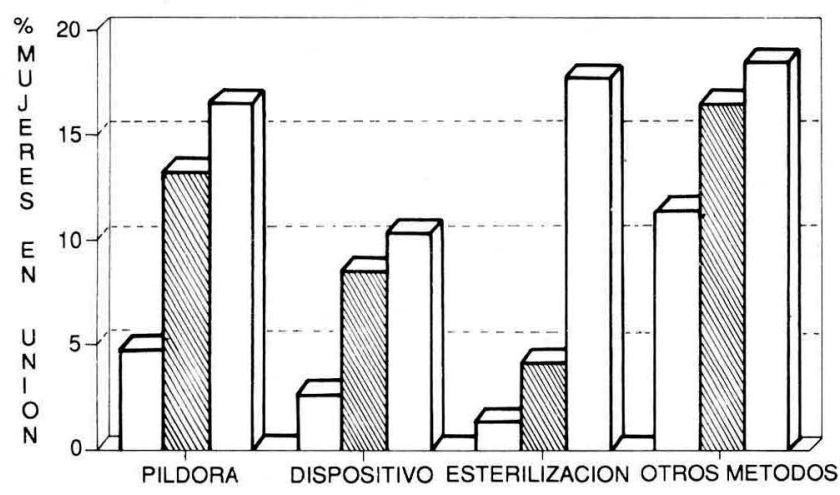

Fuente: Ministerio de Salud.

Cuadro 5

TASAS DE FECUNDIDAD, MORTALIDAD INFANTIL, ANALFABETISMO Y PRODUCTO INTERNO BRUTO (P.I.B.) POR HABITANTE, POR DEPARTAMENTOS. Censo de 1985

\begin{tabular}{|lcccr|}
\hline Departamento & $\begin{array}{c}\text { Tasa global } \\
\text { defecundidad }\end{array}$ & $\begin{array}{c}\text { Tasa aproximada } \\
\text { de mortalidad } \\
\text { infantil }\end{array}$ & $\begin{array}{c}\text { Tasa de } \\
\text { analfa- } \\
\text { betismo* }\end{array}$ & $\begin{array}{r}\text { P.I.B por } \\
\text { habitante } \\
\text { (miles } \$ \text { ) }\end{array}$ \\
\hline Antioquia & 2,7 & 49,6 & 9,8 & 186 \\
Atlántico & 2,8 & 40,0 & 8,8 & 149 \\
Bogotá & 2,2 & 31,3 & 3,9 & 263 \\
Bolívar & 3,5 & 46,3 & 20,0 & 133 \\
Boyacá & 3,5 & 40,0 & 16,8 & 117 \\
Caldas & 2,5 & 51,6 & 9,8 & 123 \\
Caquetá & 3,7 & 69,2 & 16,2 & 91 \\
Cauca & 3,6 & 57,5 & 17,5 & 87 \\
Cesar & 3,7 & 53,8 & 20,0 & 115 \\
Córdoba & 3,4 & {$[38,8]$} & 26,1 & 86 \\
Cundinamarca & 2,9 & 33,4 & 12,4 & 206 \\
Chocó & 4,5 & {$[15,0]$} & 30,6 & 73 \\
Huila & 3,6 & 49,6 & 14,0 & 183 \\
Guajira & 4,0 & {$[39,9]$} & 15,2 & 329 \\
Magdalena & 3,7 & 46,7 & 21,2 & 86 \\
Meta & 3,1 & 57,4 & 10,4 & 167 \\
Nariño & 3,3 & 73,0 & 19,3 & 71 \\
N.Santander & 3,6 & 50,1 & 16,7 & 117 \\
Quindío & 2,4 & 50,2 & 8,7 & 221 \\
Risaralda & 2,6 & 46,5 & 9,1 & 177 \\
Santander & 3,1 & 38,2 & 13,4 & 195 \\
Sucre & 3,7 & 44,2 & 28,4 & 77 \\
Tolima & 2,8 & 46,0 & 13,6 & 130 \\
Valle & 2,4 & 51,4 & 8,0 & 197 \\
\hline TOTAL & 2.9 & 46,2 & 12,2 & 169 \\
\hline
\end{tabular}

* En mayores de 15 años.

[] Subregistro evidente.

Fuente: DANE, censo de 1985.

Al igual que en los estudios de individuos (Figura 2), las tasas de fecundidad de los departamentos están altamente correlacionadas con las tasas de analfabetismo $(\mathrm{r}=0,83)(\mathrm{Fi}-$ gura 3). La correlación con el P.I.B. por habitante no es tan alta $(r=0,42)$, pero se mantiene significante (Figura 4). Las diferencias regionales en la fecundidad comienzan ahora a explicar en parte la desaceleración en el descenso de las tasas del país y surge como un desafío para los servicios de planificación familiar el llegar a las familias con más altas tasas de analfabetismo en las regiones más apartadas del país.

En Colombia PROFAMILIA es la fuente de suministro de métodos para más de la tercera parte de las usuarias de anticoncepción (Cuadro 6), sirviendo básicamente a las usuarias de DIU y esterilización. Las droguerías atienden al $30 \%$ de las usuarias, principalmente a las que utilizan anticonceptivos orales, inyecciones, preservativos y los métodos vaginales. Los hospitales y centros de salud fueron mencionados por el $15 \%$ de las mujeres entrevistadas en la ENP-86 como su principal fuente de suministro o información.

Figura 2

USO DE METODOS ANTICONCEPTIVOS COLOMBIA-1984

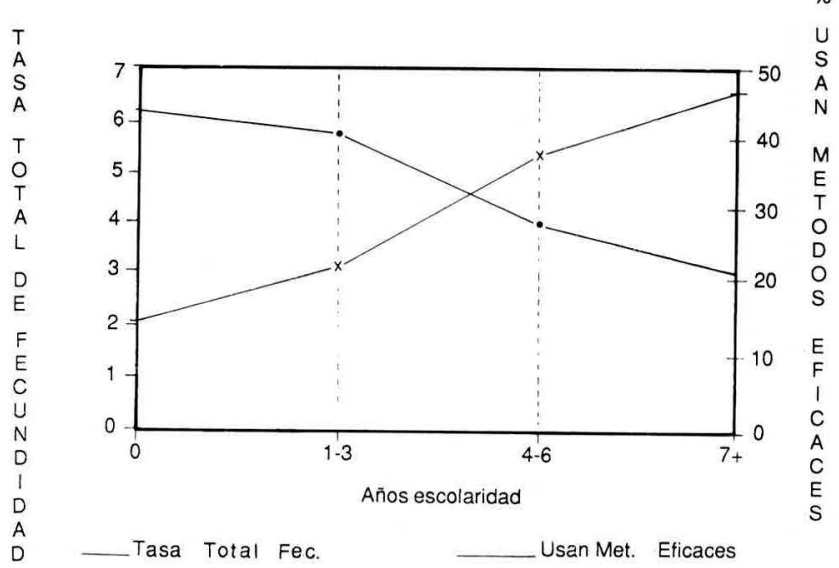

Fuente: Ministerio de Salud, 1987

Figura 3

TASA DE FECUNDIDAD VS. TASA ANALFABETISMO

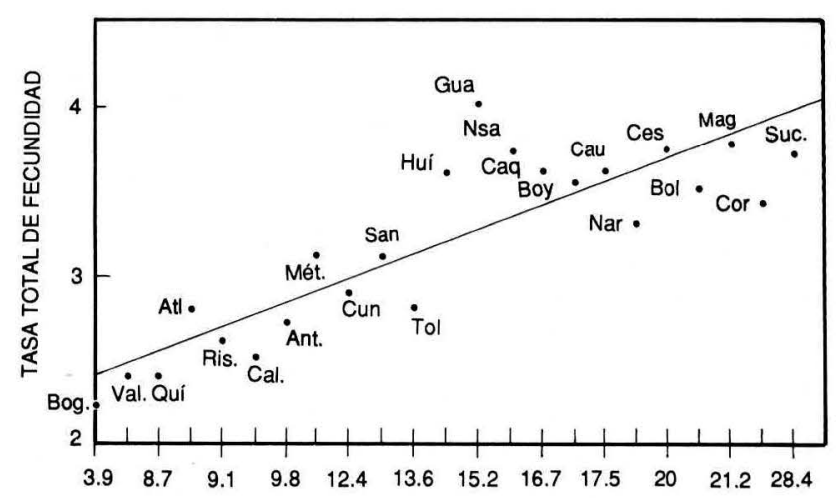

Tasa de Analfabetismo 
Cuadro 6

DISTRIBUCION PORCENTUAL DE MUJERES USUARIAS DE SERVICIOS DE PLANIFICACION FAMILIAR POR FUENTE MAS RECIENTE DE ABASTECIMIENTO E INFORMACION, SEGUN METODO, ENP-86

\begin{tabular}{|c|c|c|c|c|c|c|c|}
\hline \multirow[b]{2}{*}{$\begin{array}{l}\text { Fuente de } \\
\text { Abastecimiento } \\
\text { o Información }\end{array}$} & \multicolumn{7}{|c|}{ METODO } \\
\hline & ACO & DIU & Iny. & $\begin{array}{l}\text { Met. } \\
\text { Vag. }\end{array}$ & Pres. & $\begin{array}{l}\text { Es } \\
\text { Fem. }\end{array}$ & $\begin{array}{l}\text { ac. } \\
\text { Masc. }\end{array}$ \\
\hline Hosp/C. de S. & 9,7 & 35,5 & 8,3 & 0,0 & 3,3 & 11,9 & 0.0 \\
\hline Promotora & 0,3 & 0,0 & 2,4 & 0,0 & 0,0 & 0,0 & 0,0 \\
\hline Clin. Profamilia & 1,8 & 45,2 & 1,8 & 2,4 & 10,0 & 71,7 & 100.0 \\
\hline Puesto Profam. & 7,1 & 0,0 & 1,3 & 3,4 & 7,0 & 0,0 & 0,0 \\
\hline Clin. Privada & 0,4 & 4,7 & 3,7 & 0,0 & 0,0 & 11,2 & 0,0 \\
\hline Médico Priv. & 0,2 & 9,3 & 6,7 & 0,0 & 1,7 & 0,3 & 0,0 \\
\hline Droguería & 75,3 & 0,6 & 73,4 & 88,9 & 65,0 & 0,2 & 0,0 \\
\hline Caja Comp. Fam. & 4,0 & 4,1 & 1,1 & 3,3 & 0,0 & 4,7 & 0,0 \\
\hline Otro sitio & 0,5 & 0,6 & 0,0 & 1,1 & 1,8 & 0,0 & 0,0 \\
\hline No sabe & 0,8 & 0,0 & 1,3 & 0,9 & 11,2 & 0,0 & 0,0 \\
\hline
\end{tabular}

Fuente: CCRP-Minsalud-IRD, 1988.

Figura 4

TASA DE FECUNDIDAD VS. PBI/HABT.

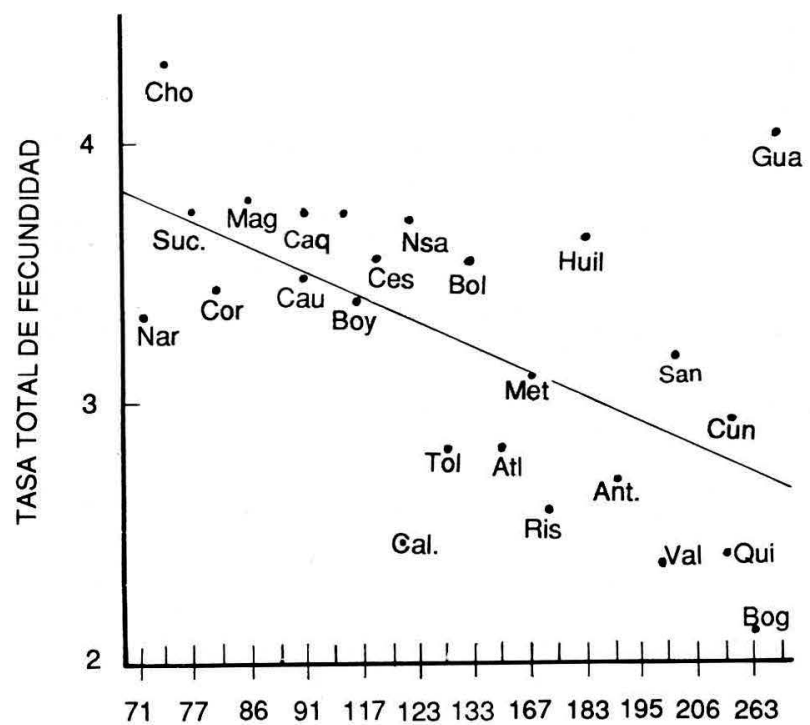

$\mathrm{PIB} / \mathrm{hbt}$ (Miles)

En cuanto a la salud de la madre, Colombia presenta tasas de mortalidad materna muy altas. Para 1964 la tasa de mortalidad materna se estimaba en 26 por 10.000 , descendiendo en 1973 a 22 y en 1984 a 11,7 por 10.000 nacidos vivos. Algunos países de la región con características socioeconómicas similares como México habían llegado a la tasa de $12 / 10.000$ en 1976 , siendo de todas maneras esta mortalidad 2 veces superior a la encontrada en Argentina, y 10 veces superior a la de los Estados Unidos de Norteamérica (6). Las causas obstétricas directas (Cuadro 7) ocurren en el $76,2 \%$ de las defunciones, seguidas por un $23 \%$ de aborto (5).

La toxemia del embarazo contribuye con un $50 \%$ de las muertes maternas por causas directas, y permanece irreductible desde 1975, lo cual amerita un análisis de la calidad de la prestación de los servicios. Aunque la cobertura de control prenatal por parte del sector oficial ha aumentado de $37,9 \%$ en 1970 a $77,1 \%$ en 1984 , la captación es tardía (último trimestre del embarazo), y el promedio de controles prenatales por personal médico es de 2,3 consultas (5). De los 2.689 nacimientos ocurridos en el grupo de mujeres entrevistadas en la Encuesta de Demografía y Salud de 1986 (7), el $61 \%$ fueron atendidas por personal médico y un $37 \%$ por personal para-médico o por algún miembro de la familia. La comadrona atiende el $30 \%$ de los partos en el área rural.

Un estudio realizado en 1986 en la Costa Caucana del Pacífico reveló una tasa de 125 defunciones maternas por 10.000 nacidos vivos (8) la cual comparada con la de la ciudad de Cali, 12/10.000, para el mismo período, apunta nuevamente hacia las grandes diferencias regionales que existen en el país.

Las estadísticas sobre aborto son prácticamente inexistentes en los años ochenta, habiéndose convertido su estudio en una especie de tabú cultural y religioso, lo cual impide la caracterización epidemiológica y el control de una de las complicaciones más letales del embarazo.

Para el aborto, una vez superado el problema tecnológico de ofrecer mejores alternativas de planificación familiar, se

Cuadro 7

'DISTRIBUCION PORCENTUAL DE LA MORTALIDAD MATERNA EN MUJERES DE 15-44 AÑOS POR CAUSAS OBSTETRICAS DIRECTAS. Colombia, 1979-1984

\begin{tabular}{|lcc|}
\hline Causa & \% 1979 & $\% 1984$ \\
\hline Toxemia del embarazo & 56.6 & 54.2 \\
$\begin{array}{l}\text { Complicaciones del } \\
\text { puerperio }\end{array}$ & 23.9 & 25.0 \\
$\begin{array}{l}\text { Hemorragia del } \\
\text { embarazo y del parto }\end{array}$ & 15.4 & 17.4 \\
$\begin{array}{l}\text { Infecciones del aparato } \\
\text { genitourinario en el } \\
\text { embarazo }\end{array}$ & 3.3 & 2.6 \\
Parto obstruido & 0.8 & 0.8 \\
\hline
\end{tabular}

Fuente: Ministerio de Salud. "Programa Materno Infantil: Hechos y Proyecciones", 1987. 
hace indispensable un planteamiento de salud pública que permita estudiarlo como complicación del embarazo, particularmente en el grupo de adolescentes que es el de más alto riesgo. El Cuadro 8 pone en evidencia la posible magnitud del subregistro en este campo, que de acuerdo con las estadísticas de los años setenta, está entre 10 y 100 veces (9).

En la ENP-86 (7) un 94,3\% de las madres entrevistadas lactaban a sus hijos durante el primer mes, $80,4 \%$ lo hacían hasta el tercer mes, y al final del noveno mes de vida un $45,2 \%$ continuaban lactando a sus hijos. Según la edad de la madre, las menores de 30 años lactaban durante 10,7 meses en promedio y las mayores de 30 lo hacían durante 11,8 meses. En el área rural los períodos promedio se extendieron hasta 13,2 meses en comparación con el área urbana, donde la duración promedio de la lactancia fue de 9,7 meses. El nivel de educación de la madre modifica la duración de la lactancia, encontrándose en la ENP-86 una duración promedio de 9,5 meses en las entrevistadas con educación secundaria, 11,9 meses en las con educación primaria y 12,4 meses en aquellas madres sin ningún grado de escolaridad. La misma ENP-86 encontró una tasa de infertilidad del 7,2\% en mujeres de 15 a 49 años, con vida sexual activa y $\sin$ práctica anticonceptiva.

Cuadro 8

TASAS DE ABORTO POR REGIONES DEL PAIS (por 100,000 nacidos vivos)

\begin{tabular}{|lc|}
\hline Región & Tasa \\
\hline Bogotá & 32.7 \\
Central & 15.9 \\
Pacífica & 20.7 \\
Atlántica & 9.8 \\
Oriental & 7.6 \\
\hline TOTAL & 15.6 \\
\hline
\end{tabular}

Fuente: Ministerio de Salud. "Programa Materno Infantil: Hechos y Proyecciones", 1987.

Los datos sobre tumores del aparato reproductivo en mujeres, para todo el país son escasos y antigüos. En 1987 el Ministerio de Salud (5) analiza las estadísticas de mortalidad de 1981, año para el cual los tumores malignos ocupan la primera posición entre las causas de muerte en mujeres de 15 a 44 años, con un $14 \%$ del total de defunciones, y una tasa ajustada de 22 por 100.000 . En este grupo de causas el cáncer de cervix uterino ocupa el primer lugar. Las estadísticas sobre enfermedades de transmisión sexual, incluyendo el Síndrome de Inmuno Deficiencia Adquirida (SIDA), son igualmente escasas e incompletas para el país.

En términos de mortalidad infantil Colombia logró un descenso del $25 \%$ en sus tasas entre 1966 y 1981, del 81,0 al 60,9 por mil nacidos vivos (10). Durante el mismo período Cali pasó del 69,6 al 27,7 por mil, un descenso del $60 \%$, pero regiones como Cauca y Nariño permanecieron estables en tasas sumamente altas (100,2 por mil). El Estudio Nacional de Salud (10) muestra cómo las diferencias en las tasas y la velocidad con que descienden, están ligadas al grado de desarrollo regional y a las diferencias educativas en la madre. En 1966 la mortalidad infantil entre los nacidos de madres sin ninguna instrucción era del orden de 103,9 por mil y descendió a 91,6 por mil en 1981 (12\%), mientras que para las madres con 6 y más años de escolaridad las tasas eran de 54,5 en 1966 y 34,2 por mil en 1981 , un descenso del $37 \%$.

La Figura 5 ilustra igualmente el aumento gradual de las coberturas de consulta ambulatoria para los menores de un año y las embarazadas.

Señala la Encuesta Nacional de Salud cómo las tasas de mortalidad infantil son más heterogéneas en 1981 que en 1966. Mientras en 1966 las distancias eran entre el 45 por mil (Bogotá) y 114 por mil (Antioquia excluyendo Medellín), en 1981 las distanciass se amplían entre 28 (Cali) y 115 por mil (Costa Pacífica rural). El DANE estima para el censo de 1985 (2) una mortalidad aproximada del 46,2 por mil nacidos vivos.

Entre 1973 y 1977 la estructura por causas de la mortalidad infantil no se modificó en el país. Las tres primeras causas de muerte que se mantuvieron en su orden, enfermedades infecciosas intestinales, enfermedad respiratoria aguda y otras perinatales, fueron responsables de la mitad de las muertes en los menores de un año. Para 1981 (5) "otras causas perinatales" ascendió al primer lugar (17,6\% de las defunciones), seguido por infecciosas intestinales (16\%) y enfermedad respiratoria aguda (13\%). Es posible que en el decenio actual se comiencen a reflejar en las tasas de mortalidad infantil los resultados de las dos estrategias más exitosas de la atención primaria en los ochentas, las campañas masivas de vacunación y las sales de rehidratación oral en el manejo de la enfermedad diarreica aguda. El Cuadro 9 ilustra el gran incremento que se logra en coberturas de vacunación entre 1980 y 1987.

En el Simposio-Taller sobre Lineamientos en Salud Reproductiva y Tecnología Diagnóstica Perinatal (Medellín, marzo 22 al 25 de 1988), el grupo de trabajo en embarazo de la adolescente calcula que hay 600.000 adolescentes expuestas a quedar embarazadas. En el Diagnóstico de Salud Reproductiva en Colombia (Ministerio de Salud, Bogotá, abril de 1988) se analizan las causas de consulta externa por causas relacionadas con el embarazo en adolescentes (12-18 años), y de un total de 22.824 consultas en 1985 , $534(2 \%)$ corresponden a embarazos terminados en aborto.

Figura 5

\section{CONSULTA MEDICA EXTERNA} SUBSECTOR OFICIAL MIXTO

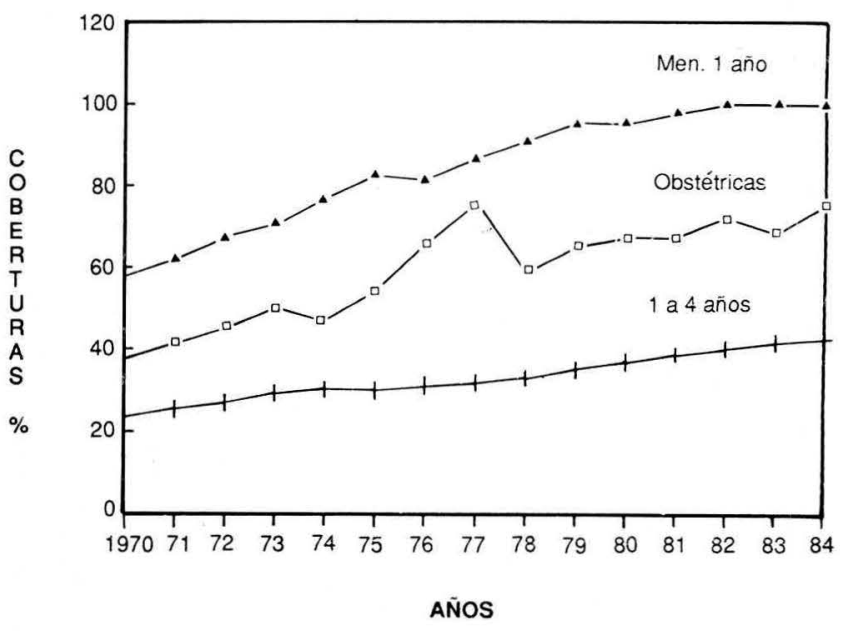

Fuente: Macroindicadores de Salud, 1984 
Cuadro 9

COBERTURA DE VACUNACION EN MENORES DE 4 AÑOS, COLOMBIA, 1980- 1987

\begin{tabular}{|lccc|}
\hline Vacuna & Grupo de edad & 1980 & 1987 \\
\hline Polio & men. 1 & 16.1 & 67.5 \\
& men. 4 & 30.5 & 86.5 \\
D.P.T. & men. 1 & 15.1 & 52.6 \\
& men. 4 & 28.1 & 75.6 \\
Sarampión & men. 1 & 13.7 & 53.4 \\
& men. 4 & 35.0 & 78.2 \\
B.C.G. & & & \\
& men. 1 & 47.0 & 72.2 \\
& men. 4 & 42.6 & 85.4 \\
\hline
\end{tabular}

Fuente: Ministerio de Salud. "Memoria al Congreso", 1987-1988.

Por causas de egreso hospitalario en el mismo año, de los 6.998 egresos en mujeres de 12 a 15 años por causas asociadas al embarazo, parto y puerperio, 511 se debieron a embarazo terminado en aborto $(7,4 \%)$ y $4.761(68 \%)$ fueron partos normales. De los 68.444 egresos por causas relacionadas con el embarazo, parto y puerperio, en mujeres de 16 a 18 años, $4.420(6,4 \%)$ se debieron a embarazo terminado en aborto y 49.650 ( $72 \%$ ) fueron partos normales.
Anexo 1

POBLACION COLOMBIANA POR EDADY SEXO, CENSO 1985

\begin{tabular}{|lrrr|}
\hline Grupo de edad & Hombres & Mujeres & Total \\
\hline Men. 1 año & 312.866 & 299.184 & 612.050 \\
$1-4$ años & 1.404 .540 & 1.353 .332 & 2.757 .872 \\
$5-9^{\prime \prime}$ & 1.750 .586 & 1.694 .262 & 3.444 .848 \\
$10-14^{\prime \prime}$ & 1.639 .319 & 1.586 .948 & 3.226 .267 \\
$15-19^{\prime \prime}$ & 1.582 .367 & 1.672 .504 & 3.254 .871 \\
$20-24^{\prime \prime}$ & 1.440 .203 & 1.560 .397 & 3.000 .600 \\
$25-29^{\prime \prime}$ & 1.151 .976 & 1.265 .155 & 2.417 .131 \\
$30-34^{\prime \prime}$ & 937.799 & 969.476 & 1.907 .275 \\
$35-39^{\prime \prime}$ & 813.010 & 851.686 & 1.664 .696 \\
$40-44^{\prime \prime}$ & 619.497 & 605.027 & 1.224 .524 \\
$45-49^{\prime \prime}$ & 514.955 & 529.154 & 1.044 .109 \\
$50-54^{\prime \prime}$ & 456.950 & 463.996 & 919.946 \\
$55-59^{\prime \prime}$ & 345.915 & 348.464 & 694.379 \\
$60-64^{\prime \prime}$ & 287.781 & 290.918 & 578.699 \\
$65-69^{\prime \prime}$ & 195.046 & 204.696 & 399.742 \\
$70 y-"$ & 325.890 & 365.033 & 690.923 \\
\hline TOTAL & 13.777 .700 & 14.060 .232 & 27.837 .932 \\
\hline
\end{tabular}

Fuente: DANE, censo 1985.

\section{BIBLIOGRAFIA}

1. Rosero L. Infant mortality decline in Costa Rica. En: Halstead SB, Walsh JA and KS Warren (eds.) "Good health at low cost". The Rockefeller Foundation, New York, 1985.

2. Departamento Administrativo Nacional de Estadística (DANE). XV Censo Nacional de Población y IV de Vivienda, Vol. I, Bogotá, 1986.

3. Prada E y Ojeda G. Primeros hallazgos de la Encuesta de Demografía y Salud de Colombia, 1986. Perspectivas Internacionales en Planificación Familiar, Número Especial de 1988.

4. Weinberg MB, Lloyd C and Blank AK. Womens's education and fertility: a decade of change in four Latin American countries. International Family Planning Perspectives 1989; 15. (1): 4-14.

5. Ministerio de Salud. República de Colombia. "Programa Materno Infantil: Hechos y Proyecciones". 3a. ed. Bogotá, 1987.

6. Mier y Teran M. Some aspects of the interrationship between fertility patterns and health. Wld Hlth Stat Quart 1987; 40. (1): 41-53.
7. CCRP, Ministerio de Salud e Institute for Resource Development/ Westighouse, "Encuesta Nacional de Prevalencia del Uso de Anticonceptivos y Primera de Demografia y Salud". Bogotá, 1988.

8. Saa Barona O. "Causas de la Mortalidad Neonatal en la Unidad Regional de Occidente", Guapi - 1987. Tesis de Magister en Epidemiología, Departamento de Medicina Social, Universidad del Valle, Cali, 1989.

9. Cusminsky M y Roberts EN. Demografía, nivel de salud y metas propuestas para el año 2000. En: "Salud Materno Infantil y Atención Primaria en las Américas: Hechos y Tendencias. Publicación Científica No. 461, Organización Panamericana de la Salud, Washington D.C., 1984

10. Bayona A y Pabón A. "La Mortalidad en Colombia", Vol II. Estudio Nacional de Salud, Minsalud-INS-Ascofame, Bogotá, julio de 1983. 